\section{Kidney \\ Blood Pressure Research}

Original Paper

\title{
The Role of CD44-Hyaluronic Acid Interaction in Exogenous Mesenchymal Stem Cells Homing to Rat Remnant Kidney
}

\author{
Xiao-Hui Bian ${ }^{a, b}$ Guang-Yu Zhou ${ }^{b}$ Li-Ning Wang ${ }^{a}$ Jian-Fei Ma Qiu-Ling Fan Na Liu ${ }^{b}$ \\ Yu Bai ${ }^{b}$ Wei Guoc Yan-Qiu Wang ${ }^{b}$ Guang-Ping Sun ${ }^{b}$ Ping He ${ }^{b}$ Xu Yang ${ }^{b}$ Xue-Song Su ${ }^{b}$ \\ Feng Du ${ }^{b}$ Gui-Feng Zhao ${ }^{d}$ Jia-Ning Miao ${ }^{d}$ Li Ma $^{e}$ Li-Qiang Zheng $^{f}$ De-Tian Li ${ }^{b}$ \\ Jiang-Min Fenga
}

a'Departments of Nephrology, The First Affiliated Hospital, China Medical University; ${ }^{b}$ Departments of Nephrology, Shengjing Hospital, China Medical University; 'The fourth hospital of People; 'Key Laboratory of Health Ministry for Congential Malformation, Shengjing Hospital, China medical

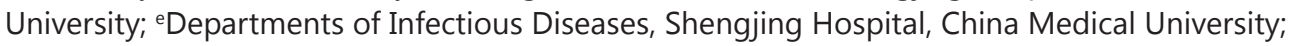
'Departments of Library, Shengjing Hospital, China Medical University, Shenyang, Liaoning, China

\section{Key Words}

Mesenchymal stem cell $•$ Hyaluronic acid $\cdot$ CD44 $\bullet$ Chronic renal failure $•$ 5/6 Nephrectomy

\begin{abstract}
Background/Aims: The aim of our study was to reveal the role of CD44-Hyaluronic acid (HA) in the homing and improving renal function of systemically transplanted MSCs in chronic renal failure. Methods: First, a remnant kidney model was established in rats and the expression of HA was determined using immunohistochemistry (IHC) and western blotting. Next, chemotaxis assay using flow cytometry, and cell migration assay of MSCs were performed in vitro. Then, MSCs were transplanted into rats, thus, sprague-Dawley (SD) rats were randomly divided into sham group, 5/6 nephrectomy (5/6 Nx) group, MSC group and MSC/Anti-CD44 group ( $\mathrm{n}=8$ for all groups). Migration of MSCs to the kidney in these rats was assessed by using cell tracking experiments, and tissue damage was evaluated by morphological analysis using Masson's trichrome staining and periodic acid Schiff staining. Results: HA was significantly observed in $5 / 6$ Nx group, but not in sham group. Meanwhile, HA was discovered induced MSCs migration remarkably $(p<0.05)$ and anti-CD44 antibody inhibited the migration significantly $(p<0.05)$ in vitro. In vivo, the GFP-MSCs were observed in MSC group and the cells reduced in MSC/ Anti-CD44 groups, especially, in the tubulointerstitium. Conclusion: Our findings reveal that CD44-HA has the potential to induce MSCs homing to injured tissue, while its effect on the ability of MSCs, improving tissue function, is not significant.
\end{abstract}




\section{Kidney \\ Blood Pressure Research}

Bian/Zhou/Wang/Ma/Fan et al.: CD44-Hyaluronic Acid Interaction

\section{Introduction}

Migration of systemic administered mesenchymal stem cells (MSCs) to injured tissue is the first step of MSCs-mediated target tissue repair [1]. Despite many papers about MSCs migration have been published, the molecular mechanisms of MSCs homing have not been fully elucidated. Chemokines are the most important factors controlling cellular migration and stromal cell -derived factor-1 $\alpha$ (SDF-1) and its specific receptor CXCR4 have been discovered to be critical in promoting the recruitment of bone marrow-derived cells to the heart after myocardial infarction and can further increase homing in the presence of injury [2]. In addition, SDF-1 is sufficient to induce therapeutic stem-cell homing to injured myocardium [3]. Xu et al. have assumed that the CXCR4/SDF-1 axis plays an important role in MSC homing due to its significant involvement in hematopoietic stem cell (HSC) homing [4]. However, unlike HSCs, CXCR4 presents at the surface of a small subset of MSCs and is unstable following ex vivo expansion [5].

Therefore, a hypothesis is suggested that other factors may be involved in the process of MSCs homing to injured tissues. CD44-hyaluronic acid (CD44-HA) interactions is suggested to play a key role in MSCs homing to renal tissue and enhance renal regeneration following acute tubular injury [6]. However, few researchers have explored CD44-HA effect on chronic renal failure. In one of our previous study, we found that MSCs administered intravenously at 8 week after 5/6 nephrectomy (5/6 Nx) were easily found in damaged renal tissue, and this phenomenon was accompanied by improved renal function. Thus based on previous findings, we hypothesized that CD44-HA interaction may also play a pivotal role in MSC homing to injured tissues in chronic renal failure model.

To test this possibility, we detected the function of CD44-HA in vitro and vivo experiments, respectively. In vitro, rat remnant kidney model was established and the expression of HA was determined using immunohistochemistry (IHC) and western blotting. In vitro, chemotaxis assay and cell migration assay of MSCs were performed. Then, MSCs were transplanted into rats, receiving the corresponding reagent, followed by MSCs migration to the kidney assessment using cell tracking experiments, and tissue damage detection by morphological analysis. Renal function was also assessed by measuring serum creatinine and urea levels.

\section{Materials and Methods}

\section{Animals}

In our present study, adult male Sprague-Dawley (SD) rats (200-250 g) were obtained from the Medical Institute Animal Center of China Medical University and maintained under a $12 \mathrm{~h} \mathrm{light} / 12 \mathrm{~h}$ dark cycle, with food and water ad libitum at the animal care facilities. All procedures were approved by the Animal Research Committee of China Medical University, Shengjing hospital.

\section{Experimental rat remnant kidney model}

The rat remnant kidney model was induced as previously described [7]. In brief, the rats were subjected to a subtotal 5/6 nephrectomy (5/6 Nx) in 2 operations performed a week apart. Rats were anaesthetized with pentobarbitone sodium ( $40 \mathrm{mg} / \mathrm{kg}$ body weight, intra-peritoneal) before operation. In the first operation, $2 / 3$ of the left kidney was resected through a frank incision, and then in the second operation, the contralateral kidney was removed. In control animals, a sham procedure was performed and these animals were subjected to the same surgical procedure and conditions, without resection. Total 16 SD rats were randomly divided into sham group and 5/6 Nx group ( $\mathrm{n}=8$ for each group). Rats were killed 8 weeks after operation, then, remnant kidneys and blood samples were collected. Remnant kidney was processed for Immunohistochemistry (IHC) and Western blotting analysis.

Immunohistochemical analysis

IHC analysis in Paraplast-embedded sections was performed as previously described [8]. Briefly, the $4-\mu \mathrm{m}$-thick slides were immunostained with the primary antibody, rabbit polyclonal antibody against HABP 


\section{Kidney \\ Blood Pressure Research}

Kidney Blood Press Res 2013;38:11-20

\begin{tabular}{l|l}
\hline DOI: 10.1159/000355749 & (C) 2014 S. Karger AG, Basel
\end{tabular}

Published online: Januar 29, 2014

www.karger.com/kbr

Bian/Zhou/Wang/Ma/Fan et al.: CD44-Hyaluronic Acid Interaction

antibody (1:50, Lifespan Biosciences, USA), followed by incubation with anti-rat secondary antibody and the peroxidase-antiperoxidase (PAP) complex. Finally, the slides were revealed using 3, 3'-diaminobenzidine (DAB). Negative controls for the immunostaining procedure were prepared by omission of the first antibody using PBS. In $10 \times 40$ fields, we randomly selected 10 fields for every section. Integrated optical density (IOD) total represented the quantity of expression of HA.

\section{Western blotting analysis}

Western blotting analysis was performed as previously described by Harlow [9]. Frozen portions of renal cortex were pulverized on dry ice and total protein was extracted. Aliquots $(20 \mu \mathrm{g})$ of proteins were mixed with sample buffer [100 mM Tris/HCl, PH 6.8, $200 \mathrm{mM}$ dithiothreitol, $4 \%$ (w/v) SDS, $0.2 \%$ bromophenol blue and $20 \%$ (v/v) glycerol], dissolved in a $12 \%$ sodium dodium sulfate-polyacrylamide gel electrophoresis (SDS-PAGE) gel, transferred to poly vinylidene fluoride (PVDF) membranes and blocked with $5 \%$ fat-free milk for $2 \mathrm{~h}$ at room temperature. Then the membranes were incubated overnight at $4^{\circ} \mathrm{C}$ with mouse monoclonal antibody to rat anti-HABP antibody (1:200, Bio-World, USA). After being thoroughly washed with Tris-buffered saline-Tween, the membranes were incubated with horseradish peroxidaseconjugated second antibody (1:2000, Beijing Zhongshan Goldenbridge Biotechnology, Beijing, China) for 2 hours at room temperature. After thoroughly washing the membranes, we detected immunoreactivity using enhanced chemiluminescence technique obtained from Perkin-Elmer, Life Science (Boston, MA). The expression level for $\beta$-actin (1:2000 dilution; Santa Cruz Biotechnology, USA) was regarded as the internal control. Results were quantitatively analyzed using FluorChem V2.0 (Alpha Innotech, San Jose, CA, USA) and gray values for all protein bands were recorded.

\section{Culture of MSC and flow cytometry}

MSCs with green fluorescent protein (GFP) of SD rats were purchased from Cyagen Biosciences (Guangzhou, China). These cells were isolated from SD rats and transfected with a GFP expressing lentiviral construct. MSCs with GFP (GFP-MSCs) have a strong capacity to expand and constitutively express GFP. Flow cytometric analysis of cell surface antigens was performed using a FACSCalibur analyzer (Becton Dickinson Immunocytometry Systems, San Jose, CA) with CellQuest software. GFP-MSCs were cultured in OriCellTM Mesenchymal Stem Cell Growth Medium at $37^{\circ} \mathrm{C}$ in $5 \% \mathrm{CO}_{2}$ according to the manufacturer's instructions (Cyagen OricellTM, China). The kit components included OriCellTM SD Rat MSCs basal medium (Cat. No. RASMX-03011- 440), 10\% MSCs-qualified fetal bovine serum (Cat. No. RASMX-05001-50), and 1\% penicillin-streptomycin and glutamine. MSCs were subcultured to 5-7 passages (P 5-7) and trypsinized. Aliquots containing $1 \times 10^{7}$ MSCs were suspended in $1 \mathrm{~mL}$ PBS and used in the present experiments.

\section{Cell migration assay}

In vitro, twenty-four transwell units were used to examine cell migration using $8-\mu \mathrm{m}$ pore polycarbonate filters (CoStar Corp, Cambridge, MA, USA) as described previously [10]. A CD44 blocking antibody (OX50, Becton Dickinson, San Jose, CA, USA) which recognizes an epitope on CD44 standard isoform that has previously been shown to significantly block CD44-HA interaction was used [11, 12]. MSC $\left(5 \times 10^{4}\right.$ cells/ well) in $\alpha$-minimum essential medium ( $\alpha$-MEM), untreated or treated previously for $30 \mathrm{~min}$ at $37^{\circ} \mathrm{C}$ with OX50 were placed in the upper chamber of the transwell unit. The $\alpha$-MEM with $0,100,200$ or $300 \mu \mathrm{g} / \mathrm{ml}$ HA (Sigma-Aldrich, St. Louis, MO, USA) was placed in the lower chamber of the transwell unit. Thus, each concentration of $\mathrm{HA}$ corresponded to two kinds of MSCs, treated or untreated with $0 \times 50$. At $37^{\circ} \mathrm{C}$ in $5 \% \mathrm{CO}_{2^{\prime}}$ all MSCs were allowed to migrate for $18 \mathrm{~h}$ through the membrane pores. After incubation, cells suspended in media in the migration chamber were gently removed by flipping out the medium. The cells adhering to the top side of the membrane were scraped with a cotton applicator and the migrated MSCs were observed using a C1 confocal microscopy (Nikon, Tokyo, Japan). Furthermore, the migrated MSCs were quantified in 5 randomly chosen fields. Values were expressed as the mean fluorescence intensity of GFP. Each experiment was conducted in triplicate and the results were the means of three independent runs.

\section{MSCs transplantation}

To evaluate the MSCs ability of migrating to remnant kidney tissue, 32 SD rats were randomly divided into 4 groups: sham group (sham-operated rats receiving MSC), 5/6 Nx group (5/6 Nx rats receiving PBS), MSC group (5/6 Nx rats receiving MSC) and MSC/Anti-CD44 group (5/6 Nx rats receiving MSC pre-incubated 


\section{Kidney \\ Blood Pressure Research}

with a CD44 blocking antibody, OX50) ( $\mathrm{n}=8$ for all groups). Then rats received the corresponding reagent, 1 mL MSCs ( $1 \times 10^{7}$ MSCs suspended in $1 \mathrm{~mL}$ PBS) or $1 \mathrm{ml}$ PBS by tail vein infusion 8 weeks after $5 / 6 \mathrm{Nx}$. Rat remnant kidneys were harvested and blood samples were collected 4 weeks after infusion according to the previous study [13]. The kidneys were perfused in situ, rapidly removed and divided into 4 segments. Two of the parts were snap frozen in liquid nitrogen and stored at $-70^{\circ} \mathrm{C}$ for later analyses. The other parts were sectioned coronally and immersed in $4 \%$ neutral-buffered formalin for 24 hours.

\section{In vivo MSC tracking}

To assess the homing of MSCs to the remnant renal tissue, fluorescent dye tracking was performed [14]. Briefly, remnant kidneys were fixed in $4 \%$ neutral-buffered formalin for $24 \mathrm{~h}$, transferred to PBS containing $20 \%$ sucrose and incubated with the reagent overnight at $4{ }^{\circ} \mathrm{C}$, then stored at $-70^{\circ} \mathrm{C}$. Moreover, frozen tissues were cut into $10-\mu \mathrm{m}$-thick sections and infused in methanol subsequently for $30 \mathrm{~min}$. Tissue sections were incubated overnight at $4^{\circ} \mathrm{C}$ with the primary anti-GFP antibody (1:5000 Earth OX, LLC, San Francisco, CA, USA). After washed with PBS, the sections were incubated with secondary antibody for $6 \mathrm{~h}$ at room temperature. Then the secondary antibody was washed and kidney sections were incubated with 4', 6-diamidino-2-phenylindole (DAPI, $5 \mu \mathrm{M}$ ). The C1 confocal microscopy (Nikon, Tokyo, Japan) was used to take images for each section, in a blinded manner, by 2 unrelated researchers. All discontinuous sections were analyzed per kidney and the total mean fluorescence intensity was calculated. Furthermore, we especially examined all MSC-GFP fluorescence intensities of glomerulus per kidney both in MSC and MSC/ OX50 groups. The MSCs fluorescence intensities of tubulointerstitial were the whole intensities subtracted that of glomeruli.

\section{Renal Function Parameters and Renal Histology}

Renal function was assessed by measuring serum creatinine and urea levels. Blood samples were collected immediately prior to sacrifice. Concentrations of serum creatinine and urea were determined using routine methods at the Department of Laboratory Medicine, Shengjing Hospital of China Medical University, Shenyang, China.

Tissue damage was evaluated by morphological analysis using Masson's trichrome staining and periodic acid Schiff (PAS) staining. Formalin-fixed, paraffin-embedded tissue sections from the kidneys were cut into 3- $\mu \mathrm{m}$-thick sections and stained with PAS and Masson's trichrome for histological analysis. The extents of glomeruli and tubulointerstitial injuries were estimated using the glomeruli sclerosis index [15] and tubulointerstitial injure index [16], respectively. All tissue samples were independently evaluated by a pathologist blinded to the treatment of the rats.

\section{Statistical Analysis}

Data were presented as mean \pm standard deviation (SD). Analysis of variance (ANOVA) was used to perform inter-group comparisons with a significance level: $\mathrm{P}<0.05$. The Statistical Program for Social Sciences, ver. 17.0 (SPSS, Chicago, IL, USA), was used for statistical analysis.

\section{Results}

Expression of HA in the renal cortex in rat remnant kidney

IHC analysis showed that HA was detectable predominantly in the interstitium of inner medulla (IM) and inner stripes of outer medulla (ISOM), but hardly in the cortex in sham rats (Figure 1A). Eight weeks after 5/6 Nx, morphologic studies showed that the most widespread glomerular changes were marked swelling, vacuolization, mesangial expansion and segmental sclerosis. The principal tubule lesions remained hypertrophy, dilatation, and interstitial fibrosis. The injured cortical renal tissue contained abundant HA, mainly secreted and expressed by hypertrophy, dilatation tubules (Figure 1B), and renal interstitium (Figure 1C). The results from western blotting of protein extracts were similar to those obtained from IHC (Figure 2). 


\section{Kidney \\ Blood Pressure \\ Research}

Fig. 1. Hyaluronic acid (HA) immunostaining in tissue samples $(\times 400)$. A: sham grou p.

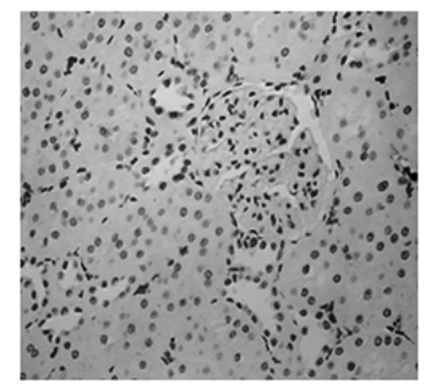

A

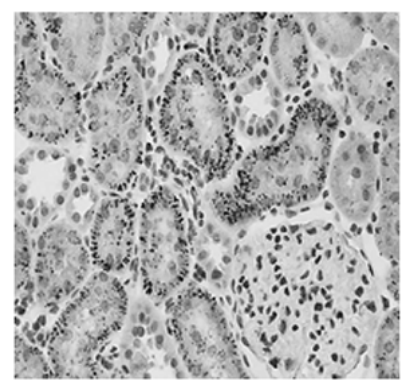

B

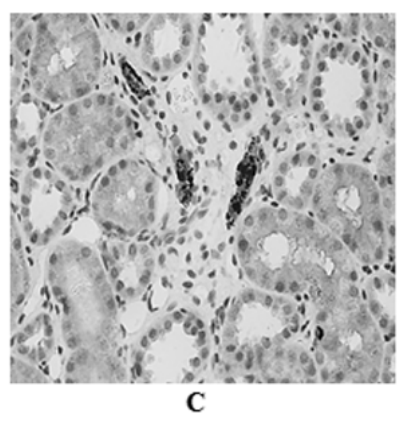

B: HA

mainly secretes in hypertrophy and dilatation tubules in 5/6 nephrectomy (5/6 Nx) group. C: HA mainly secretes in renal interstitium tubules in $5 / 6$ nephrectomy $(5 / 6 \mathrm{Nx})$ group.

Fig. 2. Western blotting of hyaluronic acid (HA) in protein extracts. C1 and C2 represent sham groups. N1 and N2 stand for $5 / 6$ nephrectomy ( $5 / 6 \mathrm{Nx}$ ) groups. $\beta$-actin is the internal control.

Fig. 3. Level of CD44 expression by mesenchymal stem cells (MSCs). This result is provided by Cyagen Biosciences (Guangzhou, China).
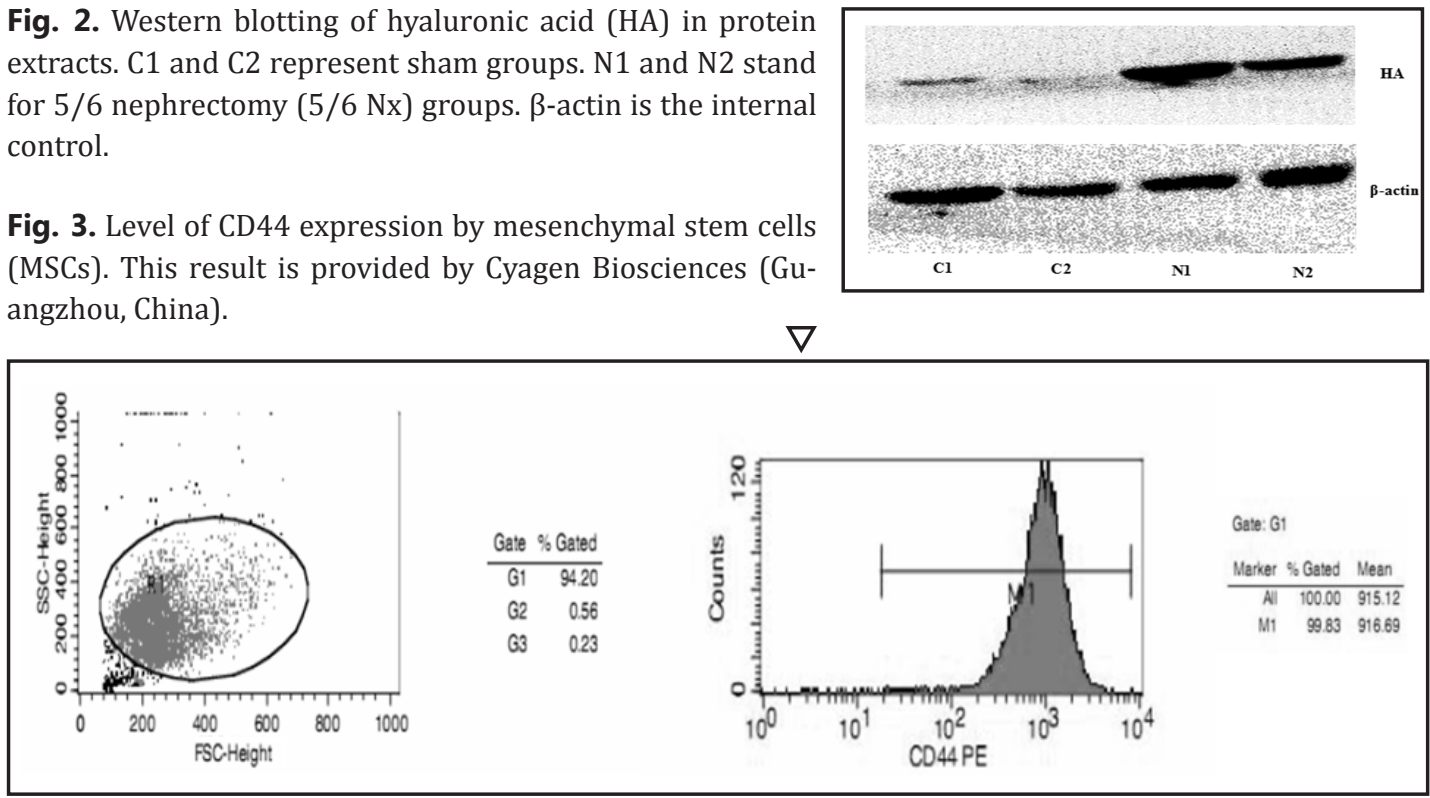

\section{HA induced a chemotactic effect on MSC}

The results of flow cytometry showed that 99.83\% MSCs expressed CD44 (Figure 3). In the in vitro chemotaxis assay, the $\alpha$-MEM without HA was used as negative control. Finally, compared with the negative control $(0 \mu \mathrm{g} / \mathrm{ml} \mathrm{HA}), 100,200$ and $300 \mu \mathrm{g} / \mathrm{ml} \mathrm{HA}$ induced the significant migration of MSCs by 224\% ( $<<0.01), 371 \%(\mathrm{p}<0.01), 157 \%(\mathrm{p}<0.01)$, respectively. Under 100, 200 and $300 \mu \mathrm{g} / \mathrm{ml} \mathrm{HA}$, the migration of MSCs pre-incubated with 0X50 was decreased by 52\% ( $<<0.01), 61 \%(\mathrm{p}<0.01), 35 \%(\mathrm{p}<0.01)$ respectively, compared with the untreated MSCs (Figure 4A-I).

\section{In vivo MSC tracking}

Four weeks after MSCs treatment, immunofluorescence analysis showed that the GFPpositive cells were only observed in rats remnant kidneys subjected to $5 / 6 \mathrm{Nx}$ (Figure 5C-E), but not in sham and 5/6 Nx + PBS groups (Figure 5A and 5B), indicating the migration of injected MSCs to the injured kidney. The MSCs labeled with GFP were observed in tubular epithelium and interstitial space. In addition, labeled cells were also found within glomeruli and peritubular capillary plexus, whereas blockage of the HA-CD44 interaction with OX50 reduced the homing by 46\% (Figures 5F-I). Furthermore, we found that there was no statistical difference of the mean fluorescence intensity of GFP in glomerulus between 


\section{Kidney \\ Bloód Pressure \\ Research}

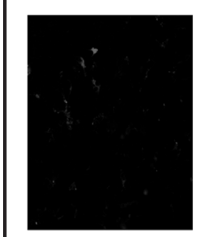

A:MSC $/(-)$

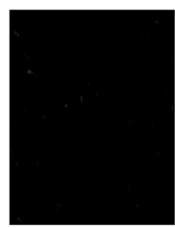

E: MSC +OX50/(-)

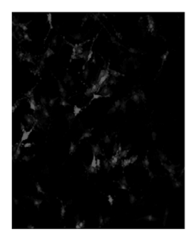

B: MSC $/ 100 \mathrm{HA}$

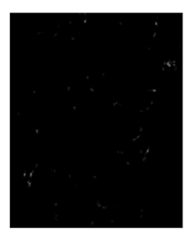

F: MSC + OX50/100HA
Bian/Zhou/Wang/Ma/Fan et al.: CD44-Hyaluronic Acid Interaction

Fig. 4. The result of migration assay $(\times 400)$. MSC, HA and OX50 are mesenchymal stem cell, hyaluronic acid and anti-CD44 antibody, respectively. ${ }^{* *} \mathrm{P}<0.01$, virus MSC $/(-)$, ${ }^{\# \# P<0.01, ~ c o m p a r e d ~ w i t h ~ M S C ~} / 100 \mathrm{HA},{ }^{\$ \$} \mathrm{P}$, virus MSC/200HA and ${ }^{\& \&} \mathrm{P}<0.01$, virus MSC/300HA.

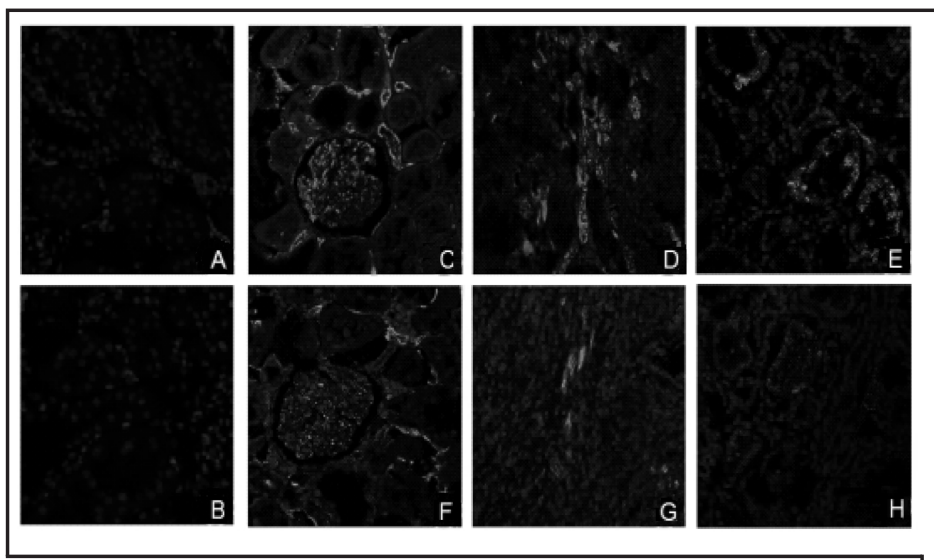

Fig. 5. Tracking MSCs labeled with GFP in remnant kidney tissue by immunofluorescence $(\times 400)$. A: sham rats treated with MSCs. B: 5/6 Nx group treated with PBS. C-E: 5/6 Nx rats treated with MSCs. F-H: 5/6 Nx group treated with MSC /anti-CD44 antibody. In addition, $\mathrm{C}$ and $\mathrm{F}$ represent glomeruli; $\mathrm{D}$ and $\mathrm{G}$ stand for renal interstitium; $\mathrm{E}$ and $\mathrm{H}$ are renal tubules. Graph I is the total fluorescence intensity in the four experimental groups and $\mathrm{J}$ is the intensity of glomerulus and tubulointerstitium. ${ }^{*} \mathrm{P}<0.05$ and ${ }^{* *} \mathrm{P}<0.01$ vs. sham group. ${ }^{\#} \mathrm{P}<0.05$ and ${ }^{\# \# P}<0.01$ vs. 5/6 Nx group. ${ }^{\&} \mathrm{P}<0.05$ vs. MSCs group.

MSC group (Figure 5C) and MSC/OX50 group (Figure 5F) (p > 0.05); however, significant differences were observed in tubulointerstitium between untreated MSCs (Figure 5D, E) and OX50 pretreated MSCs (Figure 5G, H) $(\mathrm{p}<0.05)$.

Role of MSCs in the recovery of renal injury

Compared with sham groups (Figure 6A, Figure 7A), morphologic studies showed that the most common lesions observed in rat subjected to $5 / 6 \mathrm{Nx}$ included glomerular segmental sclerosis, tubule dilatation and interstitial fibrosis (Figure 6B, Figure 7B). As compared with the 5/6 Nx group which received PBS (Figure 6A, Figure 7A), significant histological damage improvement was observed in the groups treated with MSCs (Figure 6C, Figure 7C) or MSCs/ 


\section{Kidney \\ Blood Pressure \\ Research}

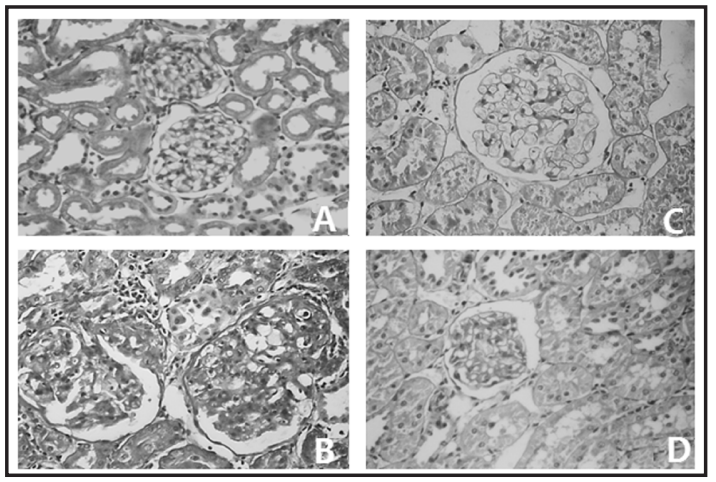

Fig. 6. Periodic acid Schiff (PAS) staining in tissue samples $(\times 400)$. A: sham group. B: $5 / 6 \mathrm{Nx}$ group. C and D represent the MSC and MSC/anti-CD44 antibody groups, respectively.

Fig. 8. Effect on renal function and histological parameters in remnant model rats with MSC or MSC pretreated with anti-CD44 antibody. A and $B$ are the serum creatinine and urea serum levels. C and D represent the indexes of glomeruli sclerosis and tubulointerstitial injuries, respectively. ${ }^{*} \mathrm{P}<0.05$ and ${ }^{* *} \mathrm{P}<0.01$ vs. sham group. ${ }^{\# P}<0.05$ and ${ }^{\# \# P}$ $<0.01$ vs. $5 / 6 \mathrm{Nx}$ group.
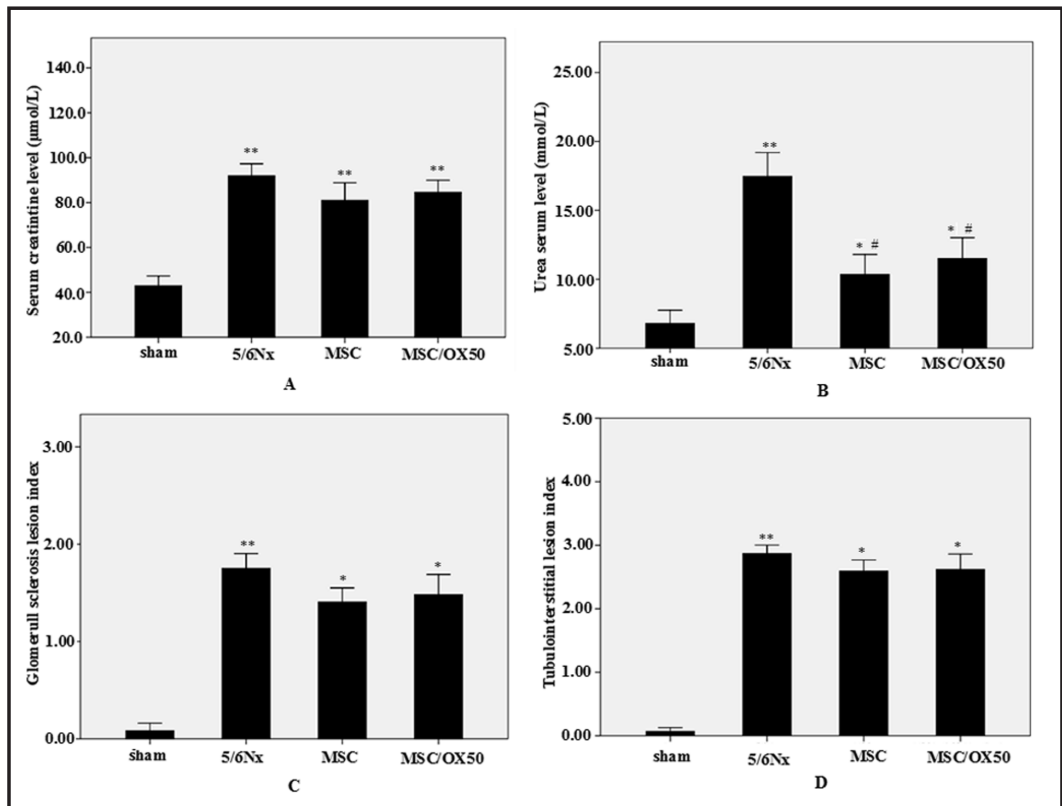

Anti-CD44 (Figure 6D, Figure 7D) at 8 weeks after 5/6 Nx. These pathologic changes were reflected by the glomerular sclerosis index and the tubulointerstitial injury index. Compared with sham group, 5/6 Nx group displayed significantly elevated blood serum creatinine and urea levels $(\mathrm{p}<0.05)$. Simultaneously, intravenous administration of MSCs significantly reduced serum creatinine and urea levels $(\mathrm{p}<0.05)$. The improvement of renal function in MSC/OX50 groups were weaker than MSC groups, but the difference was not statistically significant ( $p>0.05$ ) (Figure 8A-B). Furthermore, the differences of both indexes among four groups were similar to those obtained from renal function (Figures 8C-D).

\section{Discussion}

Migration of systemic administered MSCs to injured tissue has been demonstrated to play a crucial role in target tissue repair [17]. However, the underlying molecular mechanisms of MSCs homing to tissue have not been fully elucidated. In the migration, firstly, MSCs get the signals from the injured tissue, and then the cells migrate to the injured site. A preliminary 


\section{Kidney Blood Pressure Research}

\begin{tabular}{l|l|l}
\hline Kidney Blood Press Res 2013;38:11-20 \\
\hline DOI: 10.1159/000355749 & (c 2014 S. Karger AG, Basel
\end{tabular}

Published online: Januar 29, 2014

www.karger.com $/ \mathrm{kbr}$

Bian/Zhou/Wang/Ma/Fan et al.: CD44-Hyaluronic Acid Interaction

study has suggested that MSCs exhibit certain activities similar to HSC homing to BM under the guidance of chemical signaling navigation [18]. In our present study, the effect of CD44HA interaction on MSCs homing to injured renal tissue was studied and its potential role in renal function improvement was predicted. This study showed that CD44-HA interaction was involved in the migration of exogenous MSCs to damaged renal tissue in rat chronic renal failure model. In detail, HA was discovered to facilitate the migration potency of MSCs both in vitro and in vivo. Moreover, this migration could be inhibited by blocking with antiCD44 antibody (OX50). The membrane proteoglycan CD44 is a multifunctional signaling molecule and plays an important role in cells adhesion, migration, proliferation and differentiation [19]. More recently, compelling evidence suggests that CD44 is a key factor of the transendothelial migration of metastatic tumor cells [20]. Including endothelial cells, leukocytes and metastatic tumor cells, CD44 is also expressed in HSCs and MSCs. CD44 is the principal cell surface receptor for HA, which has been shown to accumulate in the cortex in several kidney diseases, such as I/R injury [21], chronic interstitial nephritis [22] and chronic cyclosporine nephropathy [23]. Accordingly, our data showed that HA deposition was readily detectable in remnant model (5/6 Nx). In vitro experiment, $200 \mu \mathrm{g} / \mathrm{ml} \mathrm{HA}$ significantly promoted MSCs migration and the phenomenon was suppressed by anti-CD44 antibody (OX50) remarkably. In addition, 100 and $300 \mu \mathrm{g} / \mathrm{ml} \mathrm{HA}$ also had the similar ability, but they were weaker than $200 \mu \mathrm{g} / \mathrm{ml} \mathrm{HA}$. This finding suggested that the effect of CD44-HA on MSCs migration is dose-dependent.

In addition, we also found that HA induced MSCs migration in vivo and it should be emphasized that rat remnant kidney model is appropriate for studying chronic renal failure, because it is not at all related to the initial damage to the kidney. In the present study, MSCs administered intravenously at 8 weeks post $5 / 6 \mathrm{Nx}$, when HA increased significantly, were easily observed in damaged renal tubules, the renal interstitial, glomeruli and peritubular capillaries. However, when the MSCs were pretreated with OX50, the number of MSCs migrated to the injured tissues decreased. Therefore, our data revealed that administered MSCs were poor at reaching the damaged kidneys if pre-incubated with CD44 blocking antibody, and this phenomenon was enhanced especially in damaged renal tubules and tubulointerstitium where the HA was significantly secreted. These findings suggested that HA-CD44 interaction played an important role in MSCs homing to injured kidney tissues. Similarly, the role of CD44 and HA in MSCs homing in a mouse model acute kidney injury had also been examined [6]. They found that MSCs derived from CD44-knockout mice or pre-incubated with blocking antibody did not migrate to the injured tissue. They speculated that protease-induced matrix degradation might release soluble HA fragments that could contribute to the recruitment of MSCs. Interestingly, one recent study also has showed that CD44 is involved in the homing of leukemic stem cells to their HA-rich bone marrow niche, suggesting CD44 blockade as a potential therapeutic approach in this type of cancer [24, 25].

Furthermore, we discovered that the neutralization of CD44 could not completely abolish the migration of MSC both in vivo and in vitro. This result indicated that MSCs pretreated with OX50 also have the potential to homing to injured tissue and improve its function. This result was consistent with a published paper which demonstrates that CD44\% MSCs are able to partly migrate in various degrees to HA-coated as well as uncoated chambers [26]. Based on these findings, we cannot exclude the possibility that other stimuli may also participate in the MSCs migration. One studies found that triggering of the CCL7/CCR1 and CCL2/CCR2 may play an important role in MSCs homing to the ischemic myocardium [27].

Different from a previous study [6], we unexpectedly found that MSCs pretreated with CD44 blocking antibody still could improve renal function and histological appearance, although the number of migration MSC was significantly decreased. That may be explained as followings: (1) This result might be related with the short observation time, and the observation period was prolonged, by looking for a therapeutic difference. (2) The blocking effect of antibody is stronger in vitro than in vivo. It is hypothesized that the binding of MSCs and antibody may weaken due to in vivo environment. (3) It is under debate whether engrafted MSCs have the same migratory capacity and all contribute to injured tissues with 


\section{Kidney \\ Blood Pressure Research}

the normal MSCs. It cannot be illustrated how many MSC clones are capable of sustained engraftment in targeted tissue. Recently, a cellular barcoding tool has been introduced to allow the clonal progeny of each cell to be tracked over time in the hematopoietic system [28]. By coupling the barcoding method to a sequencing-based detection system, major and minor clones in 2 distinct cell culture systems in vitro and in a long-term transplantation setting coule be identified [28]. This method provides a potential tool to study the fate of the transplanted MSCs. (4) Different large doses of MSCs, ranging from 0.4-10 $\times 10^{6}$, are currently required for successfully clinical application. However, the engraftment of MSCs in vivo is limited. It is possible that MSCs engraftment exerts favorable effects through the secretion of paracrine factors, rather than transdifferentiation. In a previous investigation, we found GFP was expressed in some renal tubular epithelial cells, peri-tubular cells, interstitial cells, and glomeruli cells. It was rational that exogenous MSCs labeled with GFP could differentiate into or fuse with renal inherent cells; however, the number of engrafted MSCs was very small. Simultaneously, MSCs transplantation could inhibit the expression of $\alpha$-SMA and TGF- $\beta 1$, even at 12 and 16 weeks after 5/6 Nx, when MSCs transplantation appeared to have no effect on renal function or histological improvement.

\section{Conclusion}

Our results suggested that CD44-HA axis might have an important role in the recruitment of injected exogenous MSCs to the injured renal tissues; however, this interaction takes unobvious effect on renal improvement in chronic renal failure.

\section{Conflict of Interest}

We have no conflict of interest to state.

\section{Reference}

1 Uccelli A, Moretta L, Pistoia V: Mesenchymal stem cells in health and disease. Nat Rev Immunol 2008;8:726-736.

2 Abbott JD, Huang Y, Liu D, Hickey R, Krause DS, Giordano FJ: Stromal cell-derived factor-1 $\alpha$ plays a critical role in stem cell recruitment to the heart after myocardial infarction but is not sufficient to induce homing in the absence of injury. Circulation 2004;110:3300-3305.

-3 Askari AT, Unzek S, Popovic ZB, Goldman CK, Forudi F, Kiedrowski M, Rovner A, Ellis SG, Thomas JD, DiCorleto PE: Effect of stromal-cell-derived factor 1 on stem-cell homing and tissue regeneration in ischaemic cardiomyopathy. Lancet 2003;362:697-703.

4 Xu J, Mora A, Shim H, Stecenko A, Brigham KL, Rojas M: Role of the sdf-1/cxcr4 axis in the pathogenesis of lung injury and fibrosis. Am J Respir Cell Mol Biol 2007;37:291.

-5 Wynn RF, Hart CA, Corradi-Perini C, O'Neill L, Evans CA, Wraith JE, Fairbairn LJ, Bellantuono I: A small proportion of mesenchymal stem cells strongly expresses functionally active cxcr4 receptor capable of promoting migration to bone marrow. Blood 2004;104:2643-2645.

6 Herrera M, Bussolati B, Bruno S, Morando L, Mauriello-Romanazzi G, Sanavio F, Stamenkovic I, Biancone L, Camussi G: Exogenous mesenchymal stem cells localize to the kidney by means of cd44 following acute tubular injury. Kidney Int 2007;72:430-441.

7 Schaefer F, Chen Y, Tsao T, Nouri P, Rabkin R: Impaired jak-stat signal transduction contributes to growth hormone resistance in chronic uremia. J Clin Invest 2001;108:467-475.

-8 Villanueva S, Cespedes C, Gonzalez A, Vio CP: Bfgf induces an earlier expression of nephrogenic proteins after ischemic acute renal failure. Am J Physiol Regul Integr Comp Physiol 2006;291:R1677-R1687. 


\section{Kidney \\ Blood Pressure Research}

\begin{tabular}{|c|c|}
\hline Kidney Blood Press Res 20 & \\
\hline $\begin{array}{l}\text { DOI: } 10.1159 / 000355749 \\
\text { Published onlIne: Januar 29, } 2014\end{array}$ & $\begin{array}{l}\text { (c) } 2014 \text { S. Karger AG, Basel } \\
\text { www.karger.com/kbr }\end{array}$ \\
\hline
\end{tabular}

Bian/Zhou/Wang/Ma/Fan et al.: CD44-Hyaluronic Acid Interaction

9 Harlow E: Lane. D.(1988) antibodies: A laboratory manual. Cold Spring Harbor Laboratory Press, Cold Spring Harbor, NY 1991;53:49-53.

10 Bourguignon LY, Zhu H, Shao L, Chen YW: Cd44 interaction with tiam1 promotes rac1 signaling and hyaluronic acid-mediated breast tumor cell migration. J Biol Chem 2000;275:1829-1838.

11 Zhu H, Mitsuhashi N, Klein A, Barsky LW, Weinberg K, Barr ML, Demetriou A, Wu GD: The role of the hyaluronan receptor cd44 in mesenchymal stem cell migration in the extracellular matrix. Stem Cells 2006;24:928-935.

12 Khaldoyanidi S, Schnabel D, Föhr N, Zöller M: Functional activity of cd44 isoforms in haemopoiesis of the rat. Br J Haematol 1997; 96:31-45.

-13 Villanueva S, Ewertz E, Carrion F, Tapia A, Vergara C, Cespedes C, Saez PJ, Luz P, Irarrazabal C, Carreno JE: Mesenchymal stem cell injection ameliorates chronic renal failure in a rat model. Clin Sci 2011;121:489499.

14 Weir C, Morel-Kopp M-C, Gill A, Tinworth K, Ladd L, Hunyor SN, Ward C: Mesenchymal stem cells: Isolation, characterisation and in vivo fluorescent dye tracking. Heart, Lung and Circulation 2008;17:395-403.

15 Aoyama I, Shimokata K, Niwa T: Combination therapy with benazepril and oral adsorbent ameliorates progressive renal fibrosis in uremic rats. Nephron 2002;90:297-312.

-16 Tanaka T, Kojima I, Ohse T, Ingelfinger JR, Adler S, Fujita T, Nangaku M: Cobalt promotes angiogenesis via hypoxia-inducible factor and protects tubulointerstitium in the remnant kidney model. Lab Invest 2005;85:1292-1307.

17 Shi Y, Hu G, Su J, Li W, Chen Q, Shou P, Xu C, Chen X, Huang Y, Zhu Z: Mesenchymal stem cells: A new strategy for immunosuppression and tissue repair. Cell Res 2010;20:510-518.

18 Wu Y, Zhao RC: The role of chemokines in mesenchymal stem cell homing to myocardium. Stem Cell Rev 2012;8:243-250.

-19 Zöller M: Cd44: Can a cancer-initiating cell profit from an abundantly expressed molecule? Nat Rev Cancer 2011;11:254-267.

20 Deboux C, Ladraa S, Cazaubon S, Ghribi-Mallah S, Weiss N, Chaverot N, Couraud PO, Baron-Van Evercooren A: Overexpression of cd44 in neural precursor cells improves trans-endothelial migration and facilitates their invasion of perivascular tissues in vivo. PloS one 2013;8:e57430.

21 Declèves A-E, Caron N, Nonclercq D, Legrand A, Toubeau G, Kramp R, Flamion B: Dynamics of hyaluronan, cd44, and inflammatory cells in the rat kidney after ischemia/reperfusion injury. Int J Mol Med 2006;18:8394.

22 Feng J, Wu J, Zhang G: The characteristics of inflammatory cell infiltration in chronic interstitial nephritis. Chinese J Nephrol 1999;6:015.

23 Han DH, Song HK, Lee SY, SONG JH, Piao SG, Yoon HE, Ghee JY, Yoon HJ, Kim J, Yang CW: Upregulation of hyaluronan and its binding receptors in an experimental model of chronic cyclosporine nephropathy. Nephrology 2010;15:216-224.

24 Jin L, Hope KJ, Zhai Q, Smadja-Joffe F, Dick JE: Targeting of cd44 eradicates human acute myeloid leukemic stem cells. Nat Med 2006;12:1167-1174.

-25 Krause DS, Lazarides K, von Andrian UH, Van Etten RA: Requirement for cd44 in homing and engraftment of bcr-abl-expressing leukemic stem cells. Nat Med 2006;12:1175-1180.

26 Rampon C, Weiss N, Deboux C, Chaverot N, Miller F, Buchet D, Tricoire-Leignel H, Cazaubon S, Evercooren BV, Couraud PO: Molecular mechanism of systemic delivery of neural precursor cells to the brain: Assembly of brain endothelial apical cups and control of transmigration by cd44. Stem Cells 2008;26:1673-1682.

-27 Yang X, Hou J, Han Z, Wang Y, Hao C, Wei L, Shi Y: One cell, multiple roles: Contribution of mesenchymal stem cells to tumor development in tumor microenvironment. Cell Biosci 2013;3:5.

28 Gerrits A, Dykstra B, Kalmykowa OJ, Klauke K, Verovskaya E, Broekhuis MJ, de Haan G, Bystrykh LV: Cellular barcoding tool for clonal analysis in the hematopoietic system. Blood 2010;115:2610-2618. 